\title{
Do environmental factors influence composition of potential allelochemicals in the submersed freshwater macrophyte Elodea nuttallii (Hydrocharitaceae)?
}

\author{
Daniela Erhard and Elisabeth M. Gross
}

\section{Introduction}

Since Elodea nuttallii (Planch.) St. John was introduced to Europe from North America in 1939 it has invaded many water bodies and is widely spread over the continent. Its success is usually related to ease of adaptation to several environmental factors (DENDĖNE et al. 1993, JONES et al. 1993, OZIMEK et al. 1993, SIMPSON 1990). Our research, however, indicates that chemical defenses may play an additional role in this process. E. nuttallii inhibited the growth of algae and cyanobacteria in laboratory bioassays, and also the growth of the aquatic herbivorous larvae of Acentria ephemerella (Lepidoptera) (ERHARD \& GRoss unpubl. results).

The production of allelochemicals causing growth reduction is often influenced by several environmental factors. The amount of allelopathically active polyphenols in Myriophyllum spicatum decreased under light limitation (GROss 2003). Both light and nitrogen limitation caused a decline of the allelopathically active fischerellin A in the benthic cyanobacterium Fischerella muscicola (GROSs et al. 1994). Which secondary metabolites in Elodea are causing the observed allelochemical effects is still unclear. During our search for these compounds, we found distinct UV-absorbing secondary metabolites in these plants, among them three flavone diglucuronides and a yet unidentified phenolic acid with similarity to caffeic acid (MuEs 1983, ERHARD et al. subm.). Flavonoids may protect plant leaves from UV radiation, herbivory, or might exert allelopathic effects (HARBORNE 1986). Their biosynthesis might also depend on light, nutrient availability or temperature. Usually, light intensity and nutrient deficiency increase flavonoid production, whereas temperature might affect the type of flavonoid produced (McClure 1986). We recently showed that E. nuttallii from various locations as well as the related $E$. canadensis produce the same flavonoid compounds, but in a distinct, species specific pattern (ERHARD et al. subm.). In accordance to MuEs (1983), we assume that the overall flavonoid production in Elodea is not affected by environmental factors. Nevertheless, differences in the total amount of individual compounds might be driven by environmental conditions.

Here, we tested whether different environmental factors influence the accumulation of four phenolic compounds in E. nuttalli by cultivating E. nuttallii under different light and temperature regimes. Based on observations that $E$. nuttallii grown in an aquarium with $\mathrm{CO}_{2}$ fertilization had higher concentrations of the yet unknown phenolic acid than plants collected in the field, we tested also the influence of $\mathrm{CO}_{2}$ availability.

Key words: allelochemicals, chemotaxonomy, secondary metabolites, invasive submersed macrophyte, phenotypic plasticity

\section{Material and methods}

In summer 2003, rooted Elodea nuttallii plants were collected in a small pond at the campus of the University of Konstanz, Germany. Ten plants harvested at the beginning of August were each planted in a transparent plastic tube, then placed in an outdoor concrete basin (mesocosm, $1 \mathrm{~m} \times 1 \mathrm{~m}$, water depth $45 \mathrm{~cm}$ ) at the Limnological Institute. Light reduction was achieved by shading five tubes with black nylon stockings. Light intensity was 600 and $1200 \mu \mathrm{E} / \mathrm{s} \cdot \mathrm{m}^{2}$ in the shaded ("MS") and uncovered ("MU") tubes, respectively. Plants taken one month later in the small pond were planted in 1.5-liter glass jars and cultivated in laboratory growth chambers (16h light/8h dark, $\left.70 \mu \mathrm{E} / \mathrm{s} \cdot \mathrm{m}^{2}\right)$ at different temperatures. Three plants were cultivated at $23^{\circ} \mathrm{C}$ ("T23"), six others at $16^{\circ} \mathrm{C}$ ("T16"). Three of the T16-plants were additionally fertilized with $\mathrm{CO}_{2}$ to investigate the influence of excess $\mathrm{CO}_{2}$ availability. Another experiment with different light conditions was conducted in October 2003. E. nuttallii were planted in glass jars and cultivated at light intensities of 130 ("LS") and 250 $\mu \mathrm{E} / \mathrm{s} \cdot \mathrm{m}^{2}$ ("LU"), respectively ( $\mathrm{n}=5$ per treatment) in a 
Table 1. Contents of the main substances in E. nuttallii, indicated as area of HPLC signals [ $\mathrm{V}^{*} \mathrm{~s}$ ], at the beginning of the experiments ("start values"). Data represent means \pm SD $(n=2-8)$. Substance 1: unidentified phenolic acid; lut, api, chry: diglucuronides of luteolin, apigenin and chrysoeriol, respectively. Different letters $(a, b)$ indicate significant differences $(P \leq 0.02)$ between sampling times.

\begin{tabular}{lccc}
\hline & August 2003 & September 2003 & October 2003 \\
\hline substance 1 & $36.98 \pm 0.39 \mathrm{a}$ & $10.07 \pm 7.12 \mathrm{~b}$ & $17.21 \pm 6.43 \mathrm{~b}$ \\
lut & $33.24 \pm 2.03 \mathrm{a}$ & $19.17 \pm 6.73 \mathrm{~b}$ & $21.12 \pm 3.68 \mathrm{~b}$ \\
api & $9.76 \pm 1.04 \mathrm{a}$ & $4.73 \pm 1.39 \mathrm{~b}$ & $5.35 \pm 0.98 \mathrm{~b}$ \\
chry & $5.11 \pm 0.73 \mathrm{a}$ & $3.73 \pm 0.87 \mathrm{a}$ & $3.80 \pm 0.64 \mathrm{a}$ \\
total & $85.09 \pm 1.32 \mathrm{a}$ & $37.70 \pm 15.52 \mathrm{~b}$ & $47.48 \pm 7.77 \mathrm{~b}$ \\
\hline
\end{tabular}

light chamber (L:D 16:8 cycle). The lower light intensities were again achieved with black nylon stockings. Experiments were run for two weeks up to two months.

To extract secondary compounds from E. nuttallii, we used cleaned, freeze-dried, finely ground plants and extracted them twice in $50 \%(\mathrm{v} / \mathrm{v})$ aqueous methanol $(1 \mathrm{ml}$ solvent per $10 \mathrm{mg}$ plant dry mass [dm]) for $2 \mathrm{~h}$ each. The crude extract was evaporated to dryness and resuspended in 50\% methanol (final concentration $100 \mathrm{mg}$ plant $\mathrm{dm}$ per $\mathrm{ml}$ ). Aliquots of these extracts equivalent to $2 \mathrm{mg} \mathrm{dm}$ were analyzed by reversed-phase high performance liquid chromatography (RP-HPLC) on a LiChrospher-100 column $(250 \times 4 \mathrm{~mm}, 5 \mu \mathrm{m})$ with a linear gradient of solvent $\mathrm{A}(1 \%[\mathrm{v} / \mathrm{v}]$ acetic acid) and B (methanol), composed of $0-40 \mathrm{~min} 5 \%$ to $60 \% \mathrm{~B}, 40-45 \mathrm{~min} 60 \%$ to $100 \% \mathrm{~B}, 45-55 \mathrm{~min} 100 \% \mathrm{~B}$. The flow rate was $1 \mathrm{ml} / \mathrm{min}$, and chromatograms were recorded at $345 \mathrm{~nm}$ using photodiode array detection. The areas of detector signals recorded at $345 \mathrm{~nm}$ from substance 1 and the flavonoids were used as quantitative measures for concentrations of these compounds in E. nuttallii. Only luteolin diglucoronide was available for calibration curves, thus no other quantification than by HPLC signal size is possible so far. Treatments were analyzed with one way ANOVA, followed by Tukey tests, when appropriate.

\section{Results}

Plants harvested in August had significantly higher amounts of secondary compounds than those sampled in September or October (Table $1)$. Thus, the contents of flavonoids and the unknown phenolic acid in E. nuttallii used in the mesocosm experiment were up to three times higher than those used in the respective laboratory experiments.

Compared to the concentrations found in freshly harvested plants, the content of substance 1 in plants cultivated in glass jars at the laboratory decreased during the experiments. This was not observed in the mesocosm experiment (Fig. 1) and resulted in significant differences between the mesocosm and laboratory treatments (one-way ANOVA, Tukey test, all pairwise comparison, $\mathrm{P}<0.02$ for $\mathrm{MU}$ and $\mathrm{P}<$ 0.03 for MS). We almost never observed an increase of any phenolic compound in the experimental treatment compared to the field plants used (ratio $\leq 1$; Fig. 1).

Substance 1 and, much weaker, apigenin and chrysoeriol diglucuronide were negatively correlated with temperature (Table 2). Substance 1 and luteolin diglucuronide slightly increased with light intensity, whereas $\mathrm{CO}_{2}$ availability, indicated by $\mathrm{pH}$, had no impact on the phenolic compounds in E. nuttallii (Table 2). A strong correlation (Pearson's $\mathrm{R}^{2}=0.71, \mathrm{P}=0.005$ ) between light intensity and peak area of luteolin diglucuronide was also observed for E. nuttallii plants collected in spring 2002 in the same pond (Fig. 2). The other substances showed no significant correlations $\left(\mathrm{R}^{2}=0.28, \mathrm{P}=0.14\right.$ for substance 1 and $\mathrm{R}^{2}=0.38, \mathrm{P}=0.08$ for the combined signal of apigenin and chrysoeriol diglucuronide).

In all plant extracts, the peak area of luteolin diglucuronide was higher than that of apigenin and chrysoeriol diglucuronide together. None of the investigated factors had any effect on the peak area ratio between luteolin and apigenin/chrysoeriol diglucuronide $\left(\mathrm{R}^{2}>0.21\right)$. Plants grown in the light chamber or in the mesocosms had higher ratios between luteolin and apigenin/chrysoeriol diglucuronide than 
Substance 1

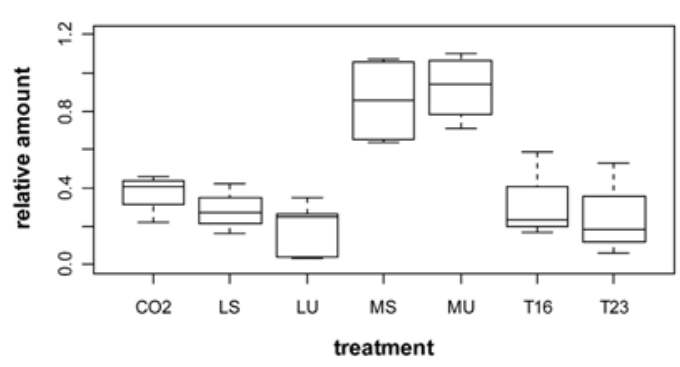

Apigenin diglucuronide

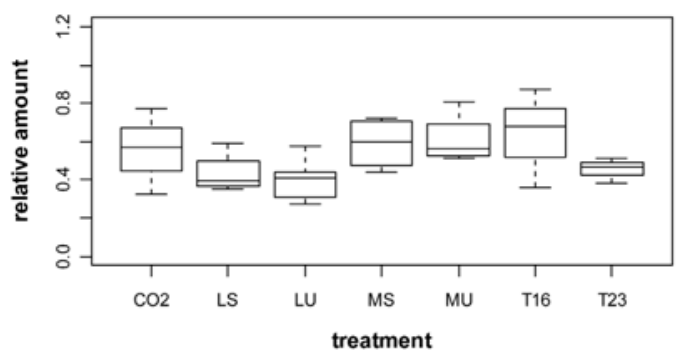

Luteolin diglucuronide

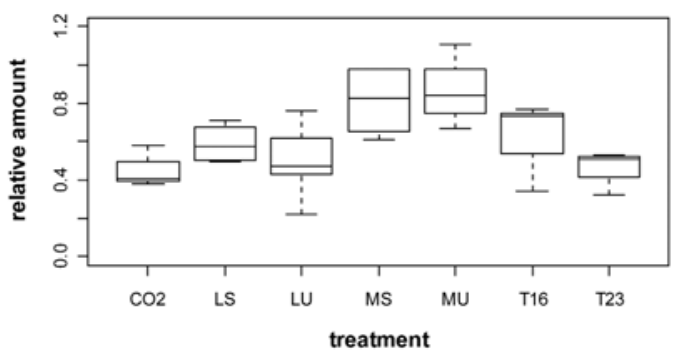

Chrysoeriol diglucuronide

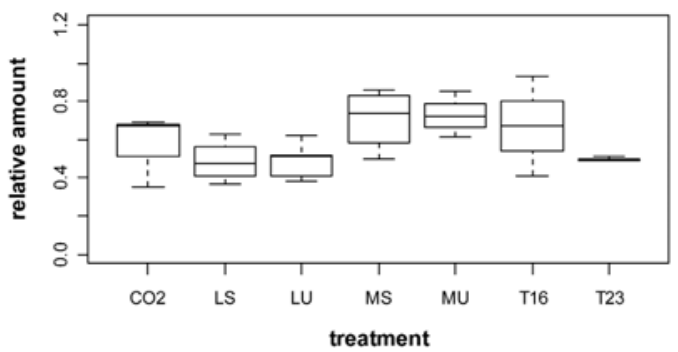

Fig. 1. Contents of secondary metabolites (in proportion to "starting values" of plants used for the respective experiments) in E. nuttallii after different cultivation experiments. $\mathrm{CO} 2: \mathrm{CO}_{2}$ fertilized plants $(n=3)$; LS: light chamber experiment, covered $(n=4)$; LU: light chamber experiment, uncovered $(n=5)$; MS: mesocosm experiment, covered $(n=4)$; MU: mesocosm experiment, uncovered $(n=4)$; T16: cultivated at ca. $16^{\circ} \mathrm{C}(\mathrm{n}=3)$; T23: cultivated at ca. $23^{\circ} \mathrm{C}(\mathrm{n}=3)$.

Table 2. Correlation of the factors temperature, light and $\mathrm{pH}$ with the four main substances in E. nuttallii extracts. Calculations are based on peak areas at the end of the experiments in proportion to starting values. Legends as in Table 1.

\begin{tabular}{lccccc}
\hline factor & substance & intercept & steepness & $\mathrm{R}^{2}$ & $\mathrm{P}$ \\
\hline temperature & substance 1 & 1.385 & -0.044 & 0.51 & $<0.0001$ \\
& lut & 1.06 & -0.021 & 0.24 & 0.015 \\
& api & 0.9556 & -0.02 & 0.36 & 0.002 \\
& chry & 1.006 & -0.02 & 0.38 & 0.001 \\
light & substance 1 & 0.2251 & 0.0006 & 0.6 & $<0.0001$ \\
& lut & 0.4937 & 0.0003 & 0.4 & 0.0005 \\
& api & 0.4774 & 0.0001 & 0.08 & 0.17 \\
& chry & 0.5217 & 0.0002 & 0.21 & 0.018 \\
$\mathrm{pH}$ & substance 1 & 0.198 & 0.024 & 0.01 & 0.632 \\
& lut & 0.310 & 0.032 & 0.04 & 0.355 \\
& api & 0.743 & -0.026 & 0.04 & 0.339 \\
& chry & 0.597 & -0.003 & 0.00 & 0.92 \\
\hline
\end{tabular}

plants used in the other laboratory experiments or plants from the pond. However, significant differences existed only between $\mathrm{LS}$ and $\mathrm{CO} 2$ $(\mathrm{P}=0.016)$ and $\mathrm{MU}$ and $\mathrm{CO} 2(\mathrm{P}=0.026)$.

\section{Discussion}

We observed considerable variations in the production of secondary metabolites in E. nuttallii. 


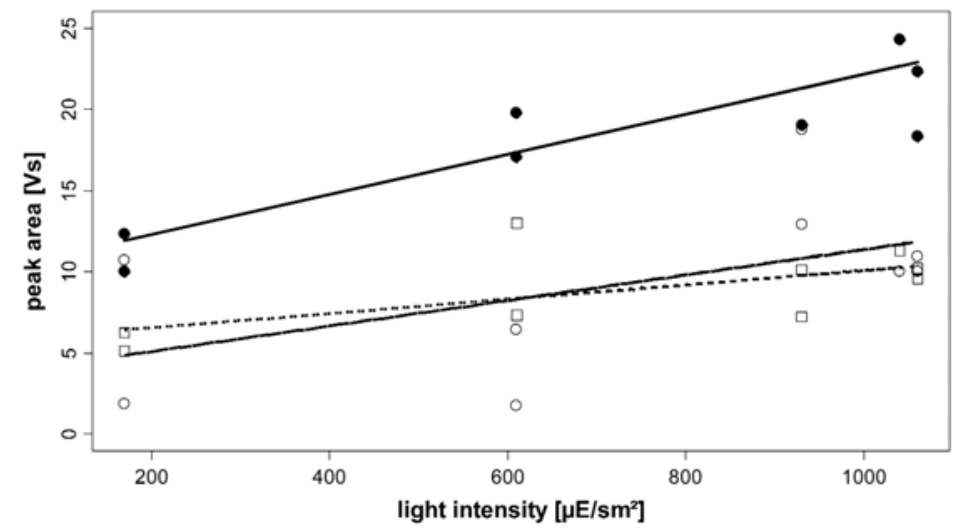

Fig. 2. Peak areas $\left[\mathrm{V}^{*} \mathrm{~s}\right]$ and regression curves of substance 1 (open circles, dashed line) and diglucuronides of luteolin (filled circles, solid line) and apigenin/chrysoeriol (squares, dotted line) in dependence of light intensity $\left[\mu \mathrm{E} / \mathrm{s} \cdot \mathrm{m}^{2}\right]$.
Even plants from the same pond may differ significantly. However, the overall pattern of flavonoids was relatively stable. We found all substances in each plant, indicating that flavonoids in Elodea are present in a general pattern and apparently not induced by environmental factors (McCluRE 1986). Since the flavonoid pattern could be used as chemotaxonomic marker, we did not expect it to vary much. Also, Mues (1983) stated that the biosynthesis of flavone glycosides depends less on external conditions. In fact, the ratio between peak areas of these substances did not fall below 1.3 and rose to maximally 3.5 . This finding is in concordance with our previous results that the peak area of luteolin diglucuronide usually exceeds that of apigenin and chrysoeriol diglucuronide (ERHARD et al. subm.).

Nevertheless, external factors might quantitatively alter individual flavonoids. The ratio between flavonoids was apparently not correlated with light intensity, temperature or $\mathrm{CO}_{2}$ availability. However, plants grown at medium or high light intensities developed higher ratios of luteolin to apigenin/chrysoeriol diglucuronides than plants cultivated at $70 \mu \mathrm{E} / \mathrm{s} \cdot \mathrm{m}^{2}$, suggesting a non-linear correlation or a threshold value. Flavonoids usually increase with light intensity, especially the glycosylated forms (MCCLURE 1986). The plant content of other phenolic compounds in the freshwater angiosperm Myriophyllum spicatum depended also on light intensity (Gross 2003). We found a positive correlation of the unknown phenolic acid and luteolin diglucuronide in E. nuttallii with light intensity. The strong correlation observed in the field supports the prediction that high irradiance might trigger luteolin diglucuronide biosynthesis. UV-B radiation increased the ratio between luteolin and apigenin (di)glucuronides in the liverwort Marchantia polymorpha (MARKHAM et al. 1998). The authors suggested that the increased level of luteolin served as scavenger of free radicals and could thus nullify the deleterious effects of UV irradiation. Another possible function of this compound may be the dissipation of absorbed UV energy in a harmless manner (MARKHAM et al. 1998). Although we did not observe a significant increase in the luteolin:apigenin ratio in E. nuttallii with light, the absolute increase of luteolin diglucuronide may be a response to higher UV-B irradiation. This would also explain the stronger correlation found in the field compared to the laboratory experiments, since laboratory cultures received artificial light with negligible UV rates.

Temperature affected all phenolic compounds except luteolin diglucuronide negatively. As indicated by the $\mathrm{R}^{2}$, these correlations explain in general less than $50 \%$ of the variability of the data, and temperature might only be one of several factors influencing the biosynthesis of secondary compounds in Elodea. However, the treatments T23, LS and LU had reduced contents of apigenin and chrysoeriol diglucuronides. These were the treatments with the highest water temperatures, which points out a temperature effect especially on these substances. 


\section{Acknowledgements}

We greatly acknowledge the help of the summercourse students KARIN FINSINGER and BIRGIT MAU. This work was supported by the German Science Foundation (DFG project no. A2 in SFB 454 to E.M.G.) and the University of Konstanz (AFF to E.M.G.)

\section{References}

Dendène, M.A., Rolland, T., TrÉMolières, M. \& CARBIENER, R., 1993: Effect of ammonium ions on the net photosynthesis of three species of Elodea. - Aquat. Bot. 46: 301-315.

Gross, E.M., 2003: Differential response of tellimagrandin II and total bioactive hydrolysable tannins in an aquatic angiosperm to changes in light and nitrogen. - Oikos 103: 497-504.

Gross, E.M., von Elert, E. \& JütTner, F., 1994: Production of allelochemicals in Fischerella muscicola under different environmental conditions. - Verh. Internat. Verein. Limnol. 25: 2231-2233.

Harborne, J.B., 1986: Nature, distribution, and function of plant flavonoids. - In: Cody, V., Middleton, E.J. \& Harborne, J.B. (Eds.): Plant flavonoids in biology and medicine: Biochemical, pharmacological, and structure-activity relationships: 15-24. Alan R. Liss, Inc., New York.
Jones, J.I., Eaton, J.W. \& Hardwick, K., 1993: Physiological plasticity in Elodea nuttallii (Planch.) St. John. - J. Aquat. Plant Manage. 31: 88-94.

Markham, K.R., RYAN, K.G., BloOR, S.J. \& Mitchell, K.A., 1998: An increase in the luteolin/apigenin ratio in Marchantia polymorpha on UV-B enhancement. Phytochemistry 48: 791-794.

McClure, J.W., 1986: Physiology of flavonoids in plants. - In: CODY, V., MidDlETON, E.J. \& HARBORNE, J.B. (Eds.): Plant flavonoids in biology and medicine: Biochemical, pharmacological, and structure-activity relationships: 77-85. Alan R. Liss, Inc., New York.

Mues, R., 1983: Species specific flavone glucuronides in Elodea species. - Biochem. Syst. Ecol. 11: 261-265.

OzimeK, T., van Donk, E. \& Gulati, R.D., 1993: Growth and nutrient uptake by two species of Elodea in experimental conditions and their role in nutrient accumulation in a macrophyte-dominated lake. - Hydrobiologia 251: 13-18.

SimPSON, D.A., 1990: Displacement of Elodea canadensis Michx by Elodea nuttallii (Planch.) H. St John in the British Isles. - Watsonia 18: 173-177.

Authors' address:

Daniela Erhard, Elisabeth M. Gross, University of Konstanz, Limnological Institute, 78457 Konstanz, Germany. E-mail: Daniela.Erhard@uni-konstanz.de 